\title{
Properties of interstellar filaments as derived from Herschel, Planck, and molecular line observations
}

\author{
Doris Arzoumanian $^{1}$, Philippe André ${ }^{2}$ and François Boulanger ${ }^{1}$ \\ ${ }^{1}$ Institut d'Astrophysique Spatiale (IAS), CNRS (UMR 8617), Université Paris-Sud 11, \\ Bâtiment 121, 91400 Orsay, France \\ ${ }^{2}$ Laboratoire AIM, CEA/DSM-CNRS-Université Paris Diderot, IRFU/Service \\ d'Astrophysique, C.E.A. Saclay, Orme des Merisiers, 91191 Gif-sur-Yvette, France
}

\begin{abstract}
Recent Herschel and Planck observations of submillimeter dust emission revealed the omnipresence of filamentary structures in the interstellar medium (ISM). The ubiquity of filaments in quiescent clouds as well as in star-forming regions indicates that the formation of filamentary structures is a natural product of the physics at play in the magnetized turbulent cold ISM. An analysis of more than 270 filaments observed with Herschel in 8 regions of the Gould Belt, shows that interstellar filaments are characterized by a narrow distribution of central width sharply peaked at $\sim 0.1 \mathrm{pc}$, while they span a wide column density range. Molecular line observations of a sample of these filaments show evidence of an increase in the velocity dispersion of dense filaments with column density, suggesting an evolution in mass per unit length due to accretion of surrounding material onto these star-forming filaments. The analyses of Planck dust polarization observations show that both the mean magnetic field and its fluctuations along the filaments are different from those of their surrounding clouds. This points to a coupling between the matter and the $\vec{B}$-field in the filament formation process. These observational results, derived from dust and gas tracers in total and polarized intensity, set strong constraints on our understanding of the formation and evolution of filaments in the ISM. They provide important clues on the initial conditions of the star formation process along interstellar filaments.
\end{abstract}

Keywords. ISM, star formation, interstellar filament formation and evolution, magnetic fields

\section{Omnipresence of filamentary structures in the interstellar medium}

While molecular clouds were already known to exhibit filamentary structures (e.g., Schneider \& Elmegreen 1979; Abergel et al. 1994; Goldsmith et al. 2008), the omnipresence of filaments in the interstellar medium (ISM) and molecular clouds has only recently been revealed thanks to the high resolution and the high dynamic range of Herschel observations of the submillimeter (submm) dust emission (e.g., André et al. 2010; Men'shchikov et al. 2010; Molinari et al. 2010). Furthermore, the all-sky maps of dust submm emission observed by Planck in total intensity, as well as in polarized intensity, emphasize the large-scale, hierarchical filamentary texture of the Galactic ISM (Planck Collaboration Int. XIX 2015; Planck Collaboration Int. XXXII 2014). These Herschel and Planck data show that interstellar matter is organized in web-like networks of filaments, which appear to be formed as a natural result of the physics at play in the ISM. Filamentary structures are also easily produced by any numerical simulation of molecular cloud formation and evolution that includes hydrodynamic or magnetohydrodynamic turbulence (e.g., Federrath et al. 2010; Hennebelle et al. 2008; Mac Low \& Klessen 2004). The presence of interstellar filaments in both star-forming regions as well as in non-star-forming quiescent clouds, alludes to a formation process of filaments preceding any star-forming activity (André et al. 2010). The spatial distribution of prestellar cores and protostars extracted 

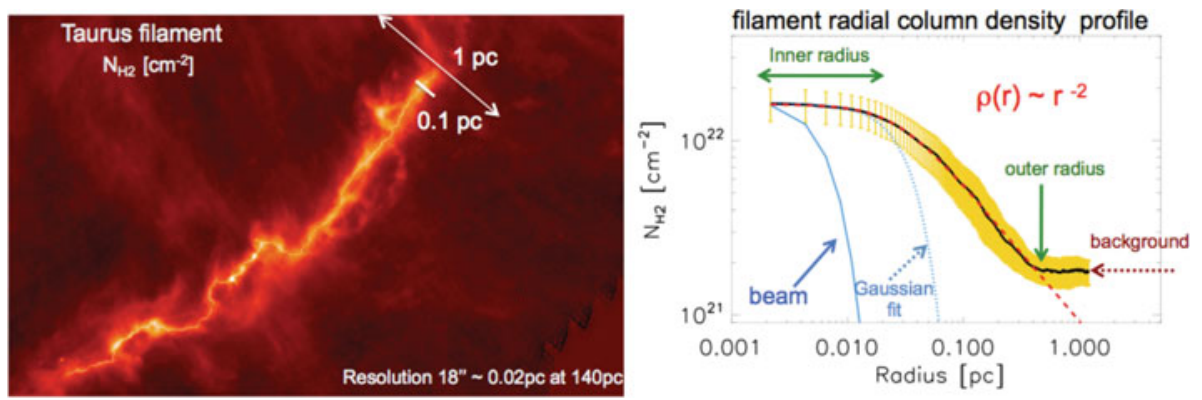

Figure 1. Example of a star-forming filament in the Taurus molecular cloud. Left: Column density map (Palmeirim et al. 2013) derived from Herschel images observed as part of the Herschel Gould Belt survey (André et al. 2010). The crest of the filament, over-plotted in white, is traced using the DisPerSE algorithm (Sousbie 2011; Arzoumanian et al. 2011). Right: Radial column density profile averaged along the filament length. The dispersion of the radial profiles along the length of the filament is shown in yellow. The profile is well fitted with a Plummer-like function (red dashed curve) where the density decreases as $r^{-2}$ at large radii (Arzoumanian et al. 2011). A Gaussian fit to the inner portion of the average column density profile yields a deconvolved FWHM width of $0.09 \pm 0.02$ pc (Palmeirim et al. 2013).

from Herschel images observed mainly on the densest filaments (Könyves et al. 2015) indicates that the properties of interstellar filaments may be a key element defining the initial conditions required for the onset of star formation (André et al. 2014).

Hence, characterizing the observed filament properties in detail, combining tracers of gas and dust in total and polarized intensities is essential to make progress in our understanding of the physical processes involved in the formation and evolution of interstellar filaments and their role in the star formation process. Herschel continuum observations are essential to describe the filament (column) density distribution. These data are complemented with ground based molecular line observations to access to the kinematics of the filamentary structures, while Planck dust polarization observations give unprecedented information on the structure of the magnetic field and its connection with interstellar matter.

In the following, I present the main results on the properties of interstellar filaments derived from Herschel, Planck, and molecular line observations. I discuss these results in the context of a new paradigm of star formation which is closely linked to the formation and fragmentation of self-gravitating filaments.

\section{Filament properties as derived from Herschel observations of nearby clouds}

Statistical analysis of nearby interstellar filaments has been possible thanks to the Herschel Gould Belt survey observations (André et al. 2010), which are ideal to characterize the filament properties, providing the resolution, the sensitivity, and the statistics (observations of a large number of clouds with different star formation activities and environments) needed for such studies.

Column density and temperature maps are derived from modified blackbody fitting of the Herschel five-wavelength images [from 70 to $500 \mu \mathrm{m}$ ] of the optically thin thermal dust emission, covering the peak of cold dust spectral energy distribution (see Fig. 1 and, e.g., Palmeirim et al. 2013). These maps are used to derive radial profiles perpendicular to the filament long axes to quantify their column density and temperature structures. The mean radial profile is derived by averaging cuts perpendicular to the crest at each pixel position along the filament (see Fig. 1). The column density profile $N_{\mathrm{H}_{2}}(r)$ is well 

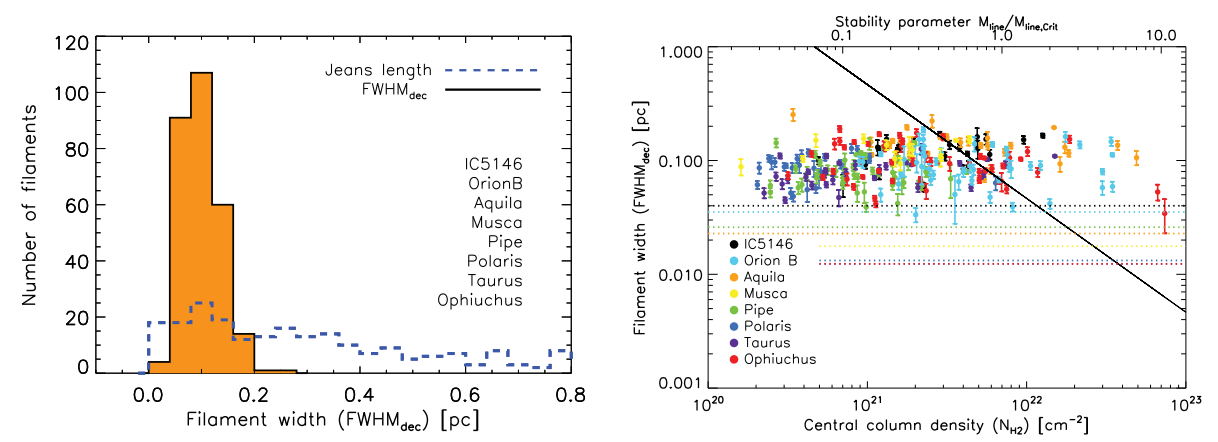

Figure 2. Left: Distribution of deconvolved FWHM widths for the 278 filaments (black solid histogram, filled in orange), with a median value of $0.09 \pm 0.04 \mathrm{pc}$. For comparison, the blue dashed histogram represents the distribution of central (thermal) Jeans lengths of the filaments. Right: Mean deconvolved width versus background subtracted central column density for the same filament sample. The spatial resolutions of the column density maps used in the analyses are marked by the horizontal dotted lines. The solid line running from top left to bottom right shows the central Jeans length as a function of central column density. The upper $x$-axis scale is an estimate of the filament mass per unit length in units of the thermal critical value $M_{\text {line,crit }}=2 c_{\mathrm{s}}^{2} / G$, where $M_{\text {line }} \propto W N_{\mathrm{H}_{2}}^{0}$ with $W=0.1$ pc (Arzoumanian et al. 2011).

fitted with a Plummer-like function of the form: $N_{p}(r)=N_{\mathrm{H}_{2}}^{0} /\left[1+\left(r / R_{\mathrm{flat}}\right)^{2}\right]^{\frac{p-1}{2}}$, where $r$ is the radial distance from the filament crest, $N_{\mathrm{H}_{2}}^{0}$ is the column density at $r=0, R_{\mathrm{flat}}$ is the radius of the flat inner part, and $p$ is the index of the corresponding density profile, which at large radii $\left(r>>R_{\text {flat }}\right)$ approaches a power law: $\rho_{p}(r) \sim r^{-p}$.

As an example, the observed profile of the Taurus B211/13 filament is shown in Fig. 1. It is characterized by a flat central part with $R_{\text {flat }}=0.03 \pm 0.01 \mathrm{pc}$ and a density decreasing as $r^{-2}$ at large radii (Palmeirim et al. 2013). The filament density profile is thus shallower than the Ostriker (1964) $\rho_{p}(r) \sim r^{-4}$ model of an isothermal filament in hydrostatic equilibrium. This observed shallower slope is also compatible with the dust temperature profile derived from Herschel observations showing a temperature drop towards the central part of the filament well described with a polytropic equation of state (Palmeirim et al. 2013). The mass per unit length $\left(M_{\text {line }}\right)$ of the filaments, estimated from their column density profiles, is a very important parameter, which defines "the stability" of a filament: a filament is subcritical, unbound when its mass per unit length is smaller than the critical value $M_{\text {line,crit }}=2 c_{s}^{2} / G \sim 16 M_{\odot} / \mathrm{pc}$ (Ostriker 1964), where $c_{\mathrm{S}} \sim 0.2 \mathrm{~km} / \mathrm{s}$ is the isothermal sound speed for $T \sim 10 \mathrm{~K}$, and $G$ is the gravitational constant. A filament is supercritical, unstable for radial collapse and fragmentation, when $M_{\text {line }}>M_{\text {line,crit }}$ (Inutsuka \& Miyama 1997).

The statistical analysis of a sample of 278 filaments observed by the Herschel Gould Belt survey in 8 regions (located at distances from 140 to $460 \mathrm{pc}$, the different regions are listed in Fig. 2) shows that the filaments span a large range in central column density and mass per unit length (Fig. 2-right), while they have a narrow distribution of FWHM widths centred around a typical value of $0.09 \pm 0.04 \mathrm{pc}$ (see Fig. 2-left, Arzoumanian et al. 2011; André et al. 2014). This characteristic filament width is well resolved by Herschel observations of the Gould Belt clouds as can be seen on the radial profile shown in Fig. 1 and Fig. 2-right, where all the measurements of the filament widths lie above the horizontal lines corresponding to the resolution limits. This typical filament width of $0.1 \mathrm{pc}$ is also in contrast with the much broader distribution of central Jeans lengths, $\lambda_{\mathrm{J}} \propto c_{\mathrm{S}}^{2} /\left(G N_{\mathrm{H}_{2}}^{0}\right.$ ), from 0.02 to $1.3 \mathrm{pc}$ (for $T=10 \mathrm{~K}$ ), implying that these filaments are not in hydrostatic equilibrium. 


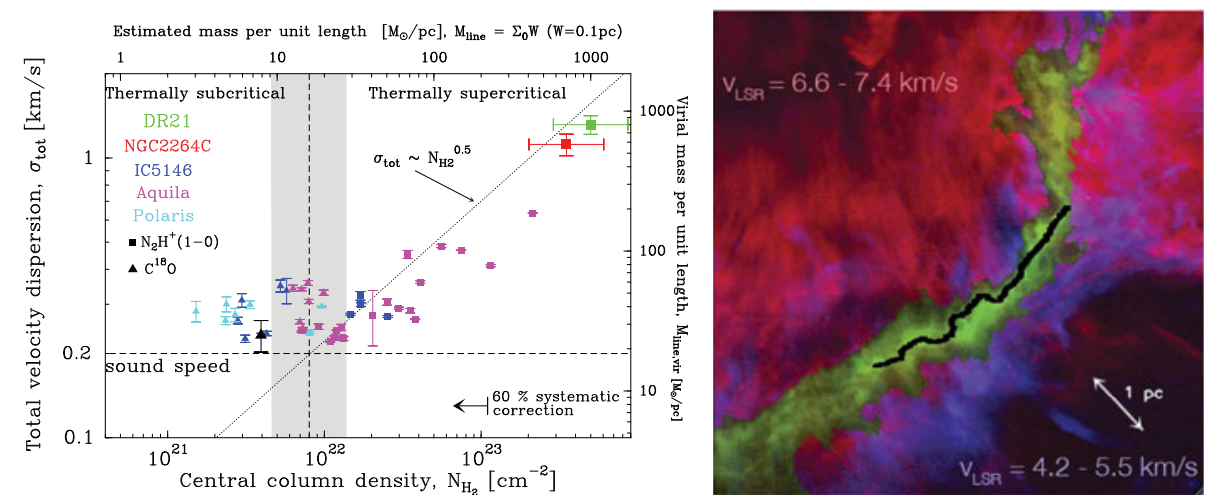

Figure 3. Left: Filament total velocity dispersion versus observed central column density. The horizontal dashed line shows the value of the sound speed $(\sim 0.2 \mathrm{~km} / \mathrm{s}$ for $\mathrm{T}=10 \mathrm{~K})$. The vertical dashed line marks the boundary between thermally subcritical and thermally supercritical filaments where the estimated mass per unit length $M_{\text {line }}$ is approximately equal to the critical value $M_{\text {line,crit }} \sim 16 \mathrm{M}_{\odot} / \mathrm{pc}$ for $\mathrm{T}=10 \mathrm{~K}$, equivalent to a column density of $8 \times 10^{21} \mathrm{~cm}^{-2}$. The grey band shows a dispersion of a factor of 3 around this nominal value. The dotted line running from the bottom left to the top right corresponds to $\sigma_{\text {tot }} \propto \mathrm{N}_{\mathrm{H}_{2}}{ }^{0.5}$ (Arzoumanian et al. 2013). Right: CO emission observed towards and around the B211/13 filament (Goldsmith et al. 2008). The systemic velocities of the ${ }^{12} \mathrm{CO}(1-0)$ emission in the north and south of the filament are redshifted and blueshifted, respectively, with respect to the velocity of the ${ }^{13} \mathrm{CO}(1-0)$ emission of the main filament shown in green (taken from Palmeirim et al. 2013). The crest of the filament is traced in black.

\section{Internal velocity dispersions of interstellar filaments derived from molecular line observations}

In the following I present molecular line spectroscopic observations of interstellar filaments and the inferred gas kinematics, which is crucial to understand the physics at play in the formation and evolution of filaments and interstellar structures.

The total velocity dispersion $\left(\sigma_{\text {tot }}\right)$ of selected positions towards a sample of 46 filaments, derived from $\left({ }^{13} \mathrm{CO}, \mathrm{C}^{18} \mathrm{O}\right.$, and $\left.\mathrm{N}_{2} \mathrm{H}^{+}\right)$molecular line observations, are presented in Fig. 3-left. Thermally subcritical and nearly critical filaments have transonic velocity dispersions $\left(c_{\mathrm{s}} \lesssim \sigma_{\text {tot }}<2 c_{\mathrm{s}}\right)$ independent of column density and are gravitationally unbound. The velocity dispersion of thermally supercritical filaments increases as a function of their column density (roughly as $\sigma_{\text {tot }} \propto \mathrm{N}_{\mathrm{H}_{2}}{ }^{0.5}$ ). These measurements confirm that there is a critical threshold in $M_{\text {line }}$ above which filaments are self-gravitating and below which they are unbound. The position of this threshold, is consistent within a factor of two with the critical value $M_{\text {line,crit }} \sim 16 \mathrm{M}_{\odot} / \mathrm{pc}$ for $\mathrm{T}=10 \mathrm{~K}$, equivalent to a column density of $8 \times 10^{21} \mathrm{~cm}^{-2}$ (Arzoumanian et al. 2013). These observations show that the mass per unit length of supercritical filaments is close to their virial mass per unit length $M_{\text {line,vir }}=2 \sigma_{\text {tot }}^{2} / G$ (Fiege \& Pudritz 2000) where $\sigma_{\text {tot }}$ is the observed total velocity dispersion (instead of the thermal sound speed used in the expression of $\left.M_{\text {line,crit }}\right)$. We suggest that the large velocity dispersions of supercritical filaments is not a result of the supersonic interstellar turbulence but may be driven by gravitational contraction/accretion (Arzoumanian et al. 2013).

Mass accretion is indirectly suggested by transverse velocity gradients observed across the self-gravitating filament in Taurus (the filament shown in Fig. 1). The systemic velocities of the observed emission in the north and south of the filament are redshifted and blueshifted, respectively, with respect to the velocity of the emission observed towards the B211/13 filament (see Fig. 3-right and Palmeirim et al. 2013). Such a velocity field 

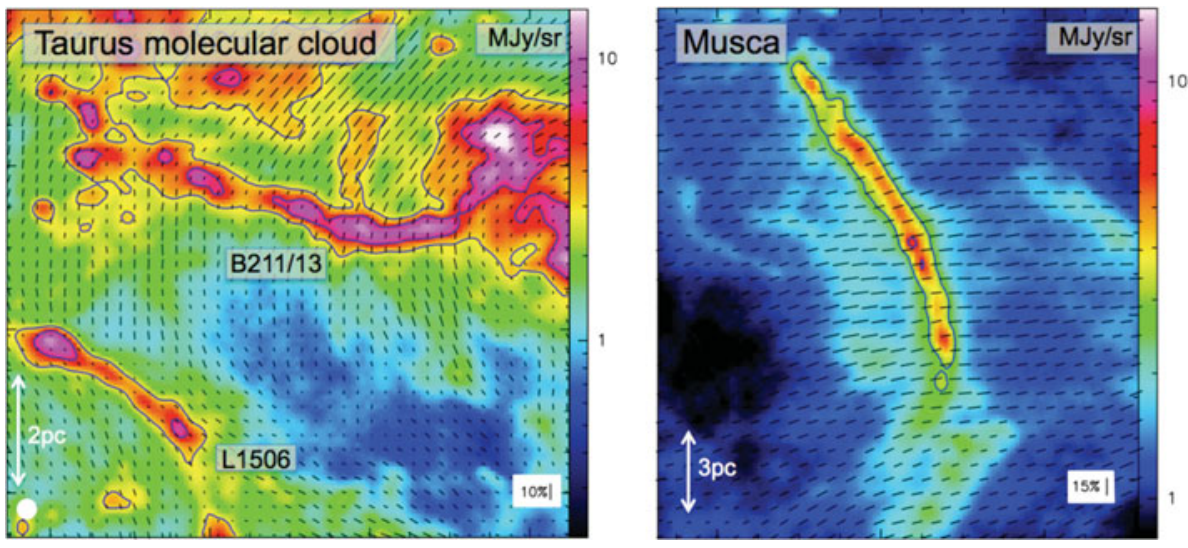

Figure 4. Planck $353 \mathrm{GHz}(850 \mu \mathrm{m})$ total dust intensity (Stokes $I$ ) maps at a resolution of 4.'8, towards the Taurus B211/13 and L1506 filaments (Left) and the Musca filament (Right). The maps are in Galactic coordinate system. The blue contours show the levels of 3 and $6 \mathrm{MJy} \mathrm{sr}^{-1}$. The black segments show the $\vec{B}_{\mathrm{POS}}$-field orientation $\left(\psi+90^{\circ}\right)$. The length of the pseudo-vectors is proportional to the polarization fraction. The polarization angles and fractions are computed at a resolution of 9.6 (indicated by the white filled circles on the left hand side map) for increased S/N (Planck Collaboration Int. XXXIII 2014).

pattern (also observed towards other filaments, e.g., Kirk et al. 2013; Lee et al. 2014; Fernández-López et al. 2014) may indicate convergence of matter onto the densest parts of supercritical filaments. This is also compatible with theoretical models for the evolution of supercritical filaments (Hennebelle \& André 2013; Heitsch 2013). Such models put forward the role of continuous accretion, which may be a physical reason to explain the observed properties of supercritical filaments.

\section{Magnetic field structure as derived from Planck dust polarization observations}

Dust polarization observations are essential to infer the orientation of the magnetic field $(\vec{B})$ component projected on the plane of the sky (POS). While the observed polarization fraction $(p)$ depends on several parameters (dust polarization properties, grain alignment efficiency, and $\vec{B}$-field structure, Hildebrand 1983; Lee \& Draine 1985), the observed polarization angle $(\psi)$ derived from dust polarized emission is perpendicular to orientation of the $\vec{B}$-field component on the POS $\left(\vec{B}_{\mathrm{POS}}\right)$ averaged along the line of sight (LOS). Planck observations at $353 \mathrm{GHz}$ provide the first fully sampled maps of the polarized dust emission towards interstellar filaments and their backgrounds, providing unprecedented insight into the $\vec{B}$-field structure.

The first striking result is the impressively ordered structure of $\vec{B}_{\mathrm{POS}}$ from the largest Galactic scales down to the smallest scales probed by Planck observations, $\sim 0.2 \mathrm{pc}$ in the nearby molecular clouds of the Gould Belt (see Fig. 4, and, e.g., Planck Collaboration Int. XIX 2015; Planck Collaboration Int. XXXIII 2014). Statistical analysis of the Planck data indicate that the relative orientation between the angle of $\vec{B}_{\mathrm{POS}}$ and that of the intensity filamentary structures changes from preferentially parallel to preferentially perpendicular from low to high column densities (Planck Collaboration Int. XXXII 2014; Planck Collaboration Int. XXXV 2015). These results confirm the rough equipartition between turbulent and magnetic energies in the ISM (e.g., Myers \& Goodman 1988) and emphasize the important role that the magnetic field plays in shaping the cold ISM structures.

To quantify the polarized intensity observed towards the filaments we derive radial profiles perpendicular to their crests and averaged along their length (as explained in 
Polarization angle across the filaments (perpendicular to the major axis)

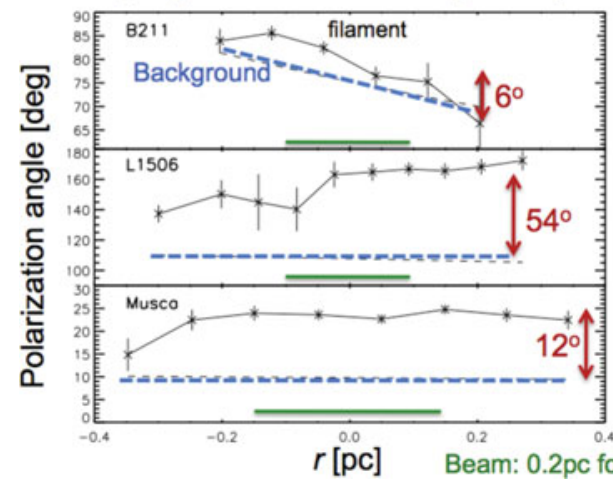

Polarization fraction across the Musca filament

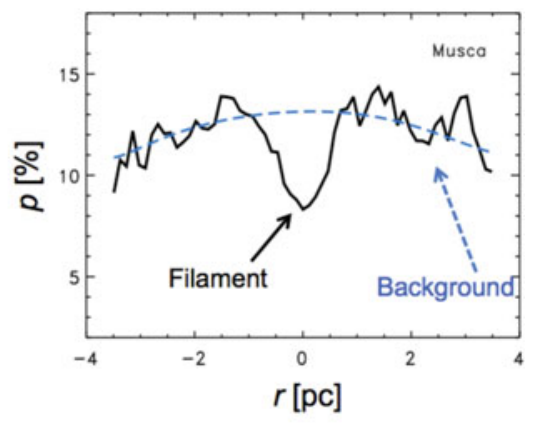

Figure 5. Left: Filament intrinsic polarization angle (tracing the angle of $\vec{B}_{\mathrm{POS}}$ ) across the crests of the B211, L1506, and Musca filaments. The $x$-axis shows the radial distance from the filament crest. The crosses are data points computed from $Q$ and $U$ background subtracted maps. The dashed line represents the background polarization angle. The difference between the polarization angle of the filament and that of the background is indicated on the plots (taken from Table 2 in Planck Collaboration Int. XXXIII 2014). Right: Observed profile (in black) of the polarization fraction $(p)$ perpendicular to the crest of the Musca filament. The dashed blue curve shows the polarization fraction of the background. It is derived from the Stokes $I, Q$, and $U$ parameters of the background.

Sect.2) to increase the signal-to-noise ratio $(\mathrm{S} / \mathrm{N})$ in polarization, while keeping the highest resolution (4.'8) of the Planck data. To derive the intrinsic polarization properties of the filaments we describe the observations as a two-component model that uses the spatial information in Planck images to separate the emission of the filaments from that of their background (i.e., surrounding cloud). This allows us to characterize and compare the polarization properties of each emission component: the filament and its background. This is an essential step in measuring the intrinsic polarization fraction $(p)$ and polarization angle ( $\psi$, which is perpendicular to the plane of the sky $\vec{B}$-field angle) of each emission component. The analyses show that both the polarization angle and fraction measured at the intensity peak of the filaments (before background subtraction) differ from their intrinsic values (after background subtraction) as described in Planck Collaboration Int. XXXIII (2014). The left panel of Fig. 5-left shows the profile of $\psi$ across the Musca, B211, and L1506 filaments. In all three cases, we measure variations in the polarization angle intrinsic to the filaments $\left(\psi_{\text {fil }}\right)$ with respect to that of their backgrounds $\left(\psi_{\mathrm{bg}}\right)$. These variations are found to be coherent along the pc-scale length of the filaments. The differences between $\psi_{\text {fil }}$ and $\psi_{\mathrm{bg}}$ for two of the three filaments are larger than the dispersion of the polarization angles across and along the filaments (see Table 2 in Planck Collaboration Int. XXXIII 2014). Hence, these differences are not random fluctuations and they indicate a change in the orientation of the POS component of the magnetic field between the filaments and their backgrounds.

The observations show a decrease in the polarization fraction $p$ from the background to the Musca filament (Fig. 5-right), as well as towards the Taurus B211 and L1506 filaments (see Fig. 10 of Planck Collaboration Int. XXXIII 2014). A decrease in $p$ with the total column density $N_{\mathrm{H}}$ (i.e., from the backgrounds to the filament crests), has been already shown in previous studies. This decrease has been usually interpreted as due to the turbulent component of the field and/or variations of dust alignment efficiency with increasing column density (e.g., Jones et al. 2015; Whittet et al. 2008). In our study, the bulk of the drop in $p$ within the filaments cannot be explained by random fluctuations 
of the orientation of the magnetic field because they are too small $\left(\sigma_{\psi}<10^{\circ}\right)$. We argue that the observed drop in $p$ towards the filaments may be due to the $3 \mathrm{D}$ structure of the magnetic field: both its orientation in the POS and with respect to the POS (Planck Collaboration Int. XXXIII 2014). Indeed, the observed changes of $\psi$ are direct evidence of variations of the orientation of the POS projection of the magnetic field. The systematic variations of $\psi$ suggest changes of the angle of the magnetic field with respect to the POS. This angle must statistically vary as much as $\psi$, contributing to the observed decrease of $p$ in the filaments. The observed variation of $\psi$ between the filaments and their backgrounds always depolarizes the total emission, due to the integration of the emission along the LOS of two emission components where the angle of $\vec{B}_{\mathrm{POS}}$ varies (see Fig. 5-right and Planck Collaboration Int. XXXIII 2014).

The inner structure of the filaments (as seen with Herschel, cf., Palmeirim et al. 2013 for B211, Ysard et al. 2013 for L1506, and Cox et al. 2015 for Musca) is not resolved, but at the smallest scales accessible with Planck ( $\sim 0.2 \mathrm{pc}$ towards the nearby clouds), the observed changes of $\psi$ and $p$ (derived from Planck polarization data at the resolution of $4 ! 8$ ) hold some information on the magnetic field structure within filaments (Planck Collaboration Int. XXXIII 2014). They show that both the mean field and its fluctuations in the filaments are different from those in the background clouds, which points to a coupling between the matter and the $\vec{B}$-field in the filament formation process.

\section{Summary and conclusions}

The ubiquity of filaments in both quiescent clouds and active star-forming regions, where they are associated with the presence of prestellar cores and protostars (Könyves et al. 2015), supports the view that filaments are (first) form in the ISM and the densest of them fragment into star-forming cores (André et al. 2014). The observational finding of a filament uniform $0.1 \mathrm{pc}$ width (Arzoumanian et al. 2011) sets very strong constraints on the physics at play in the ISM. This result has been recently confirmed by an independent analysis of the same data from another group (Koch \& Rosolowsky 2015) and observed (at higher-resolution) in regions farther away than the Gould Belt (Hill et al. 2012). Interestingly $0.1 \mathrm{pc}$ corresponds to the sonic scale below which interstellar turbulence becomes subsonic in diffuse, non-star-forming gas (Heyer et al. 2009; Pineda et al. 2011). This occurrence, along with the observed thermal velocity dispersion of low column density filaments, suggests that large-scale turbulence may be a main player in the formation of the filamentary web observed in molecular clouds (Arzoumanian et al. 2011). On the other hand, the increase of the non-thermal velocity dispersion of supercritical, self-gravitating filaments with column density, may indicate the generation of internal turbulence due to gravitational accretion. This may be an explanation for the observed constant width of self-gravitating collapsing filaments (Arzoumanian et al. 2013).

While the dissipation of interstellar turbulence provides a plausible mechanism for filament formation, the observed organization between the magnetic field lines and the intensity structures, derived from the analysis of Planck data, indicates that the $\vec{B}$-field plays a dynamically important role in shaping the interstellar matter (Planck Collaboration Int. XXXIII 2014; Planck Collaboration Int. XXXV 2015). In particular, they may be a key element to understand the channel of mass flows in the ISM. The fact that most prestellar cores lie in dense, self-gravitating filaments (Könyves et al. 2015) suggests that gravity is a major driver in the evolution of supercritical filaments and their fragmentation in star-forming prestellar cores. The detailed analysis of the distribution of matter along the filaments (density fluctuations) may also yield insights into the origin of the shape of the mass distribution of prestellar cores thought to be linked to that of the stars (André et al. 2014; Roy et al. 2015). The combination of these observational 
results, derived from dust and gas tracers in total and polarized intensity, give strong constraints on our understanding of the formation and evolution of filaments in the ISM, which provides important clues to the initial conditions of the star formation process along supercritical filaments.

\section{Acknowledgements}

DA acknowledges support by the European Research Council grant MISTIC (ERC267934).

\section{References}

Abergel, A., Boulanger, F., Mizuno, A., \& Fukui, Y. 1994, ApJ, 423, L59

André, P., Di Francesco, J., Ward-Thompson, D., et al. 2014, in Protostars and Planets VI, p. 27

André, P., Men'shchikov, A., Bontemps, S., et al. 2010, A\&A, 518, L102

Arzoumanian, D., André, P., Didelon, P., et al. 2011, A\&A, 529, L6

Arzoumanian, D., André, P., Peretto, N., \& Könyves, V. 2013, A 6 A, 553, A119

Cox, N., Arzoumanian, D., André, P., et al. 2015, A\&\&A submitted

Federrath, C., Roman-Duval, J., Klessen, R. S., Schmidt, W., \& Mac Low, M. 2010, A\&A, 512, $\mathrm{A} 81+$

Fernández-López, M., Arce, H. G., Looney, L., et al. 2014, ApJL, 790, L19

Fiege, J. D. \& Pudritz, R. E. 2000, MNRAS, 311, 85

Goldsmith, P. F., Heyer, M., Narayanan, G., et al. 2008, ApJ, 680, 428

Heitsch, F. 2013, ApJ, 769, 115

Hennebelle, P. \& André, P. 2013, A\&A, 560, A68

Hennebelle, P., Banerjee, R., Vázquez-Semadeni, E., Klessen, R. S., \& Audit, E. 2008, A\&̊A, 486, L43

Heyer, M., Krawczyk, C., Duval, J., \& Jackson, J. M. 2009, ApJ, 699, 1092

Hildebrand, R. H. 1983, QJRAS, 24, 267

Hill, T., Andre, P., Arzoumanian, D., et al. 2012, A\&A, 548, L6

Inutsuka, S. \& Miyama, S. M. 1997, ApJ, 480, 681

Jones, T. J., Bagley, M., Krejny, M., Andersson, B.-G., \& Bastien, P. 2015, AJ, 149, 31

Kirk, H., Myers, P. C., Bourke, T. L., et al. 2013, ApJ, 766, 115

Koch, E. W. \& Rosolowsky, E. W. 2015, MNRAS, 452, 3435

Könyves, V., André, P., Men'shchikov, A., et al. 2015, A\&A in press [ArXiv:1507.05926]

Lee, H. M. \& Draine, B. T. 1985, ApJ, 290, 211

Lee, K. I., Fernandez-Lopez, M., Storm, S., et al. 2014, ArXiv e-prints

Mac Low, M. \& Klessen, R. S. 2004, Reviews of Modern Physics, 76, 125

Men'shchikov, A., André, P., Didelon, P., et al. 2010, A\&SA, 518, L103+

Molinari, S., Swinyard, B., Bally, J., et al. 2010, A\&\&A, 518, L100

Myers, P. C. \& Goodman, A. A. 1988, ApJ, 326, L27

Ostriker, J. 1964, ApJ, 140, 1056

Palmeirim, P., André, P., Kirk, J., et al. 2013, A\&GA, 550, A38

Pineda, J. E., Goodman, A. A., Arce, H. G., et al. 2011, ApJl, 739, L2

Planck Collaboration Int. XIX. 2015, $A \& A$, in press [ArXiv:1405.0871]

Planck Collaboration Int. XXXII. 2014, $A \mathscr{E} A$, in press [ArXiv:1409.6728]

Planck Collaboration Int. XXXIII. 2014, $A \mathscr{E} A$, in press [ArXiv:1411.2271]

Planck Collaboration Int. XXXV. 2015, $A \mathscr{E} A$, in press [ArXiv:1502.04123]

Roy, A., André, P., Arzoumanian, D., et al. 2015, A\&A, in press [ArXiv:1509.01819]

Schneider, S. \& Elmegreen, B. G. 1979, ApJS, 41, 87

Sousbie, T. 2011, MNRAS, 414, 350

Whittet, D. C. B., Hough, J. H., Lazarian, A., \& Hoang, T. 2008, ApJ, 674, 304

Ysard, N., Abergel, A., Ristorcelli, I., et al. 2013, A\&\&A, 559, A133 